\title{
Comprensión de las dinámicas de relación de parejas del mismo sexo a partir de la intervención clínica sistémica*
}

\section{Comprehension of the Relational Dynamics of Same-Sex Couples Based on a Systemic Model for Clinical Intervention}

Recepción: 09 Abril 2012 | Aprobación: 23 Enero 2017

\author{
Carolina Herrera Small ${ }^{\mathrm{a}}$ \\ Liberarte, Colombia
}

ORCID: http://orcid.org/0000-0001-9241-8794

SimÓn TORRES Orozco

Liberarte, Colombia

a Autor de Correspondencia, Correo electrónico: info@liberarte.co

Para citar este artículo: Herrera Small, C., \& Torres Orozco, S. (2017). Comprensión de las dinámicas de relación de parejas del mismo sexo a partir de la intervención clínica sistémica. Universitas Psychologica, 16(1), 1-16. http://dx.doi.org/10.11144/J averiana.upsy16-1.cdrp

\footnotetext{
RESUMEN

Este artículo surge de una investigación que intentó comprender las dinámicas relacionales de parejas del mismo sexo que acuden a terapia y proponer un modelo de intervención clínica desde el enfoque sistémico. Se revisaron las premisas constructivistas y los conceptos de pareja, terapia de pareja y diversidad sexual. La metodología de investigaciónintervención incluyó la revisión de 98 historias clínicas y el análisis del proceso terapéutico con seis parejas. Se definieron dos tipos de parejas con características distintas en cinco ejes asociados a la emergencia del conflicto conyugal. El modelo terapéutico desarrollado requiere una evaluación completa de la dinámica conyugal que conecte el motivo de consulta y proponga estrategias para el posicionamiento de los miembros de la pareja frente a las posibilidades de la relación.

Palabras clave

diversidad sexual; psicología; pareja; sistémico; orientación sexual.

\begin{abstract}
This article is based on a study that sought to understand the relational dynamics of same-sex couples that attend therapy and to develop a systemic model for clinical intervention. Constructivist premises and concepts regarding couple dynamics, couple therapy and sexual diversity were reviewed. The methodology of this interventive research with a mixed design included the review of 98 clinical charts and the analysis of the therapeutic process with 6 couples. Two different couple profiles were established according to their predominant features regarding five analytic areas associated with couple conflict. The therapeutic approach developed requires a complete evaluation of couple dynamics, in order to connect the reason for consultation and to introduce interventive strategies that promote a clear positioning of each couple member about other relational alternatives.

Keywords

sexual diversity; psychology; systemic; sexual orientation.
\end{abstract}

| Universitas Psychologica | Colombia | V. i6 | No. i | Enero-Marzo | 20 I7 | ISSN i657-9267 | 


\section{Introducción}

Ante todo, vale la pena aclarar la utilización de ciertos términos a lo largo de este artículo y las comprensiones que la sustentan. El término "parejas del mismo sexo" refleja una comprensión compleja de las posibilidades variadas para la formación de dichas parejas a partir de miembros gais, lesbianas, bisexuales o que no se identifican con estas categorías. El término "orientación sexual" hace referencia a la atracción sexoafectiva por personas del mismo sexo, del otro sexo o de ambos sexos. Finalmente, la sexualidad se refiere a los aspectos erótico-amorosos y de afiliación que son fluidos a lo largo de la vida y van más allá de la genitalidad.

A continuación, se describen las características contextuales que justifican este estudio y los objetivos del mismo, el marco epistemológico y paradigmático asumido y los referentes relevantes sobre parejas del mismo sexo e intervención terapéutica con parejas.

Actualmente, la diversidad sexual es un tema que genera interés en múltiples disciplinas, incluyendo la psicología, en tanto la mayor visibilidad de personas, parejas, familias $y$ comunidades que asumen posturas distintas a la heteronormativa pone en duda los paradigmas y las conceptualizaciones sobre las dinámicas de relación vigentes (García, 2009). A nivel internacional, se han evidenciado en los últimos años avances legales que garantizan los derechos de personas LGBT (lesbianas, gais, bisexuales y transgénero). En 118 países, incluyendo Colombia, se ha despenalizado la homosexualidad y el matrimonio homosexual se ha legalizado en 18 países. Además, la adopción por parte de parejas del mismo sexo ya se ha legalizado en 20 países (International Lesbian, Gay, Bisexual, Trans and Intersex Association, 2015). En Colombia, a partir de las sentencias de la Corte Constitucional las parejas del mismo sexo tienen los mismos derechos que las parejas heterosexuales, incluyendo el matrimonio civil y la adopción (Colombia Diversa, 2016a). Sin embargo, las transformaciones culturales han sido más lentas, pues aún se legitiman prácticas sociales de discriminación de la diversidad sexual (Colombia Diversa, 2016b).

Es así como el objetivo de este estudio, referido a comprender las dinámicas de relación de parejas del mismo sexo que acuden a asesoría psicológica en la ciudad de Bogotá y proponer un modelo de abordaje para estas parejas desde la intervención clínica sistémica, resulta relevante, en tanto se requieren otros referentes para responder a sus necesidades particulares. Estudios anteriores han propuesto algunas directrices generales para el trabajo terapéutico con personas LGBT (Ardila, 2007; Sanders, 2009; Asociación de Psicología de Puerto Rico, 2008), pero no recogen las dinámicas particulares de la intervención clínica con parejas del mismo sexo desde el enfoque sistémico y la mayoría tampoco desarrollan análisis desde la metodología de investigaciónintervención. Además, la mayoría de estos estudios comparan las dinámicas heterosexuales con las homosexuales (Blair \& Holmberg, 2009; Diemer, Mackey, \& O'Brien, 2004; Gottman et al., 2003; Ossana, 2000), lo cual es importante pero insuficiente para comprender a fondo las dinámicas y los procesos terapéuticos con dichas parejas.

Teniendo en cuenta que existen referentes comunes a todas las modalidades de vinculación humana, se pretendió lograr una caracterización de estas parejas, comprendiendo sus particularidades sin asumir sus dinámicas como idénticas a las identificadas en parejas heterosexuales, sino partiendo de la hipótesis de que surgirían nuevas categorías comprensivas.

En cuanto al marco epistemológico y paradigmático asumido, esta investigación se inscribe en las cosmovisiones y formas de construir el conocimiento propias de un paradigma ecosistémico que sienta sus bases en la epistemología constructivista, en tanto se reconoce el papel fundamental de las relaciones interpersonales y sociales en la co-construcción de los significados, las prácticas sociales y las formas de relación (Maturana, 1995).

Desde este marco epistemológico, se estructura el proceso terapéutico de forma estratégica bajo un lente complejo más allá de la respuesta emocional de los/as 
consultantes, asumiendo la responsabilidad de influir directamente sobre ellos/as a partir de su solicitud de ayuda, y teniendo en cuenta que el cambio terapéutico de segundo orden debe atravesar cualquier comprensión e intervención (Hernández, 2004). Desde la terapia estratégica breve, los problemas que son motivo de consulta se mantienen por la interacción de los consultantes con su entorno significativo, y el objetivo de cualquier estrategia terapéutica es activar las diferentes dimensiones del cambio, tales como las experiencias emocionales, los marcos de referencia, las reglas y pautas de interacción conyugal, los marcadores de contexto, la identidad, la conciencia y la intencionalidad (Hernández, 2004).

En cuanto a las parejas del mismo sexo, desde la psicología se han estudiado temas como su vulnerabilidad de acuerdo con los marcos legales vigentes y la discriminación social que sufren (Hamrin, Prather, Riggle, \& Rostosky, 2006; Herek, 2006), las dinámicas de relación y la satisfacción de sus miembros con la misma (Connolly, 2004; Frederickson et al., 2003), así como sus estrategias de afrontamiento en contextos heterocentristas (Andrews \& Long, 2007; Beougher, Chakravarty, Darbes, Hoff, \& Nelands, 2010; Brodnicki et al., 2005). Sin embargo, se evidencia un vacío en el conocimiento científico sobre las parejas del mismo sexo y sobre abordajes terapéuticos útiles que se ajusten a sus necesidades y que trasciendan los intereses "reparativos" (McNair \& Spitalnick, 2005).

\section{Método}

\section{Diseño}

El diseño metodológico de esta investigaciónintervención incluyó el análisis cuantitativo de las historias clínicas de 98 personas que consultaron el servicio de asesoría psicológica en LIBERARTE ${ }^{[1]}$ por dificultades en su relación de pareja, así como el análisis cualitativo de los procesos terapéuticos desarrollados con seis parejas del mismo sexo, tres de mujeres y tres de hombres. El método de muestreo utilizado fue no probabilístico por conveniencia.

\section{Participantes}

En este estudio participaron 30 parejas del mismo sexo y 38 personas homosexuales y bisexuales de 20 a 52 años de edad, de estrato socioeconómico medio-alto que consultaron entre octubre de 2008 y abril de 2011 el servicio de asesoría psicológica en LIBERARTE por dificultades en su relación de pareja. La participación en la investigación fue voluntaria e informada.

\section{Instrumentos}

La información cuantitativa de las características demográficas de los participantes, así como los datos de la dinámica de la relación de pareja y del abordaje terapéutico, se registraron por escrito en las historias clínicas y fueron luego analizados mediante la prueba estadística chi-cuadrado con el paquete de análisis estadístico SPSS. El análisis de esta información se consolidó en una matriz categorial de doble entrada que permitió establecer una caracterización de las parejas que consultaron y orientar la construcción del modelo de abordaje.

Por otra parte, la información cualitativa de las sesiones de terapia de pareja fue grabada en audio y luego transcrita, manteniendo el anonimato de los/as consultantes. La información que arrojó el análisis a través de matrices categoriales corresponde a las tendencias predominantes que se presentaron en las parejas participantes y a las dimensiones de la intervención psicoterapéutica que dan cuenta del modelo de abordaje propuesto.

\section{Procedimiento}

El análisis cuantitativo inicial de las historias clínicas permitió hacer una primera descripción de las características sociodemográficas de las parejas del mismo sexo que acuden a consulta en cuanto a sus edades, el conocimiento familiar 
de sus orientaciones sexuales y la convivencia en pareja, así como de su dinámica relacional. Con este referente se orientaron los procesos terapéuticos de pareja. La caracterización de la dinámica relacional y del abordaje terapéutico se realizó mediante la formulación de ocho categorías interaccionales deductivas, las cuales se fueron afinando en sus tendencias principales a lo largo del estudio y de las cuales surgen los cinco ejes de análisis presentados en los resultados.

\section{Resultados}

A continuación, se presenta una síntesis de los resultados de las dos partes que componen este estudio: (1) los referidos a la revisión de las historias clínicas, los cuales son de carácter cuantitativo; (2) los referidos al análisis cualitativo del proceso terapéutico con parejas del mismo sexo.

En primera medida, se presentan los cinco ejes de análisis que dan cuenta de la conformación y la consolidación de las parejas del mismo sexo asociadas al conflicto. Luego se presentan otras dimensiones de la dinámica conyugal que también se asocian al conflicto de pareja. Con base en estas categorías, se definen dos grupos de parejas con características predominantes distintas, asociadas a la emergencia del conflicto conyugal. Finalmente, se presentan las características de la propuesta de abordaje para dichas parejas, teniendo como ejes articuladores el motivo de consulta, las dimensiones relevantes de la dinámica de la relación y los efectos de la intervención.

\section{Ejes de análisis}

Los cinco ejes de análisis siguen un criterio cronológico de la conformación y la consolidación del vínculo de pareja, el cual corresponde también a la organización de la dinámica de pareja dentro del abordaje terapéutico propuesto:

(1) Conformación de la relación y vicisitudes que favorecen la emergencia del conflicto: circunstancias en las que surge y avanza la relación de pareja, asociadas con la emergencia del conflicto actual: (a) el inicio en una relación triangular; (b) la tendencia a mantener triángulos en la relación; (c) las inseguridades con respecto a la relación desde su inicio; (e) las relaciones heterosexuales previas; (f) la relación a distancia; (g) la fusión de la pareja en tiempo y actividades.

(2) Motivos para permanecer en la relación: pueden ser explícitos o implícitos y se relacionan con: (a) motivos económicos; (b) la aceptación familiar de la relación; (c) compartir el proyecto de vida; (d) que es la primera relación seria; (e) compañía; (f) satisfacción sexual; (g) necesidades emocionales; (h) miedo a la soledad; (i) amenazas de suicidio de la pareja; (j) amenazas de acusaciones al jefe.

(3) Fuentes de tensión: cambios del ciclo vital, relaciones conflictivas y eventos estresantes de carácter individual que contribuyen a alimentar y mantener el conflicto conyugal: (a) la pérdida del trabajo; (b) el conflicto con la familia política; (c) el conflicto con la familia de origen; (d) el conflicto con la orientación sexual propia o de la pareja; (e) las dificultades económicas; (f) la salida de la universidad; (g) el conflicto con los hijos; (h) la infidelidad de un miembro de la pareja; (i) el conflicto con los tiempos compartidos; (j) el cambio de vivienda; (k) el cambio de ciudad; (l) la salida del closet; (m) las diferencias culturales y sociales.

(4) Motivo de consulta: definición por parte de los/as consultantes de la situación que está generando dificultades en la relación de pareja y los/as ha llevado a asistir a terapia: (a) las peleas o conflictos constantes entre los miembros que no se resuelven; (b) la ruptura o amenaza de ruptura constante de la relación, la cual de todos modos continua; (c) los celos a partir de inseguridades sobre el vínculo en uno o ambos miembros; (d) la infidelidad; (e) la mala comunicación; (f) el maltrato físico y psicológico al interior de la pareja; (g) la toma de decisiones; (h) el fortalecimiento individual.

(5) Efectos de la intervención: avances y cambios evidenciados por los/as consultantes y los terapeutas a partir del proceso terapéutico: (a) un aumento en la confianza mutua; (b) una mejor 
comunicación; (c) la aceptación de diferencias individuales; (d) la toma de decisiones sobre la continuidad o no de la relación y sobre la postura frente a la propia vida; (e) la definición de la relación a partir de la explicitación del compromiso conyugal, sus posibilidades y sus limitaciones; (f) el cese de las interacciones violentas; (g) la interrupción de la pauta predominante de la relación; (g) la disminución de las peleas en número e intensidad; (h) el reconocimiento de las fortalezas individuales.

Otras dimensiones de la dinámica de pareja que se asocian al conflicto:

(6) Pauta predominante en la relación: pauta que conecta las interacciones de la pareja, siendo las tendencias más llamativas (a) la ambigua, la cual se presenta cuando hay dudas en los miembros de la pareja sobre el compromiso con la relación que no se explicitan ni se resuelven a través de una comunicación abierta sobre las necesidades de cada uno/a, sino que alimentan patrones de relación predominantes de maltrato o chantaje emocional para comprometer al/a la otro/a, proyectando en la pareja la responsabilidad de la propia satisfacción y (b) la simbiótica, referida a la fusión entre dos personas que se sienten incompletas y no pueden funcionar sin la presencia psicológica o real del otro, donde un miembro asume el liderazgo y la protección del cónyuge para cumplir metas comunes, mientras el otro muestra una apariencia de fragilidad que le impide tomar las riendas sobre su vida. Cualquiera de los dos puede sentir agotamiento al no recibir el reconocimiento emocional esperado o al no querer posponer sus proyectos personales por el chantaje emocional del cónyuge que se muestra frágil o dependiente de la relación de pareja (Brown \& Woollams, 1979).

(7) Creencias y valores: marco de referencia personal, compuesto por creencias, posturas y valores: (a) religiosos; (b) heteronormativos; (c) homofobia internalizada; (d) vulnerabilidad al expresar emociones; (e) la convivencia legitima la relación.

(8) Reglas de la relación: acuerdos explícitos o implícitos entre los miembros de la pareja sobre el funcionamiento de la relación en cuanto a (a) roles de género culturales y convencionales; (b) distribución de tareas; (c) el manejo de la economía.

Caracterización de dos grupos de parejas con pautas predominantes asociadas al conflicto

Siguiendo los ejes de análisis definidos, a continuación, se definen dos grupos de parejas con características predominantes distintas, asociadas a la emergencia del conflicto conyugal.

\section{Figura 1}

Caracterización del grupo de parejas con pauta predominante ambigua (grupo 1).

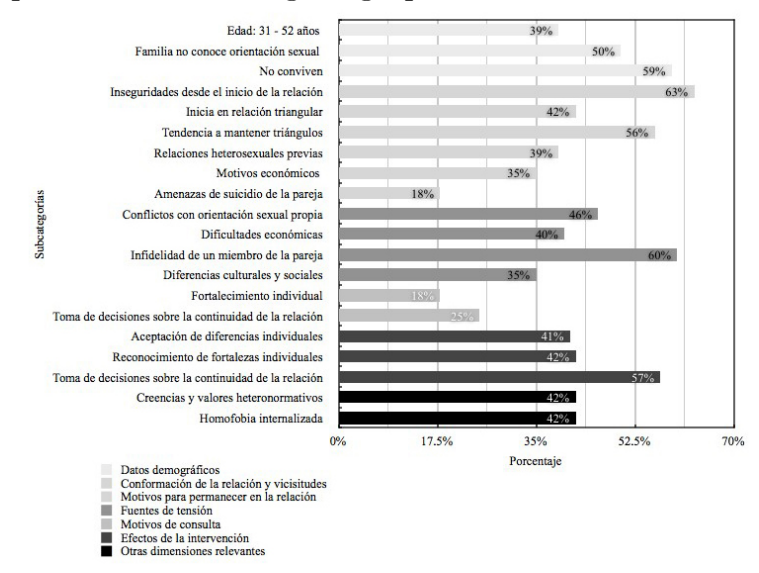

Fuente: elaboración propia.

La Figura 1 muestra que en el grupo 1 la conformación de la relación se asocia a inseguridades desde el inicio, a un inicio en relación triangular, a la tendencia a mantener triángulos y a relaciones heterosexuales previas. Los motivos para permanecer en ella son económicos o relacionados con amenazas de suicidio de la pareja. Las principales fuentes de tensión son los conflictos con la orientación sexual, las dificultades económicas, la infidelidad de un miembro y las diferencias sociales y culturales entre los miembros. En este escenario, los principales motivos de consulta son el fortalecimiento individual y la toma de decisiones sobre la continuidad de la relación. Finalmente, los efectos de la intervención en este grupo fueron la toma de decisiones sobre la continuidad de la relación, el reconocimiento de las fortalezas individuales y la aceptación 
de diferencias individuales. Otras dimensiones de la dinámica relacional que contribuyen a la caracterización de este grupo se refieren a una mayor tendencia a los valores heteronormativos y a la homofobia internalizada, así como una tendencia a mantener una pauta predominante ambigua. Finalmente, estas dinámicas de pareja se dan entre miembros con edades entre los 31 y los 52 años que no conviven y cuyas familias no conocen su orientación sexual.

En síntesis, se trata de parejas en las que sus miembros tienen conflictos individuales no resueltos relacionados con su orientación sexual, fundamentados en creencias heteronormativas y en una homofobia internalizada, lo cual los/as lleva a conformar relaciones de pareja confusas y triangulares en las que se dificulta el ajuste inicial a partir de inseguridades de los miembros e interferencias de terceros. Estas relaciones avanzan con tensiones individuales y propias de la relación y se mantienen por motivos obligatorios más que opcionales, ante lo cual es comprensible que los motivos de consulta estén relacionados con una necesidad de asumir una postura individual más clara y poder tomar decisiones frente a la continuidad de la relación.

\section{Figura 2}

Caracterización del grupo de parejas con pauta predominante simbiótica (grupo 2).

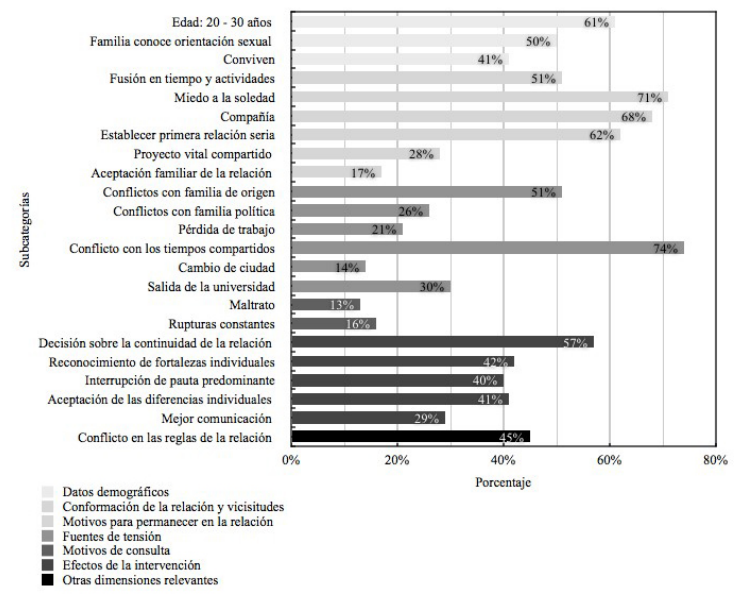

Fuente: elaboración propia.

Como se observa en la Figura 2, en el grupo 2 la conformación de la relación se encuentra asociada a la fusión de tiempo y actividades.
Los motivos para permanecer en la relación se refieren al miedo a la soledad, la compañía, el deseo de establecer la primera relación seria, el compartir el proyecto vital y la aceptación familiar de la relación. Las principales fuentes de tensión hacen referencia a los conflictos con la familia de origen y política, la pérdida del trabajo, el conflicto con los tiempos compartidos, el cambio de ciudad y la salida de la universidad. En este escenario, donde las fuentes de tensión son familiares y externas y en el que se cuenta con la relación de pareja como única fuente de apoyo, resulta comprensible que el aferramiento emocional lleve a dinámicas de maltrato o de rupturas constantes, las cuales constituyen los principales motivos de consulta. Finalmente, los efectos de la intervención se refieren a la toma de decisiones sobre la continuidad de la relación, el reconocimiento de las fortalezas individuales, la interrupción de la pauta predominante de la relación, la aceptación de las diferencias individuales y una mejoría en la comunicación.

En cuanto a las otras dimensiones de la dinámica de la pareja que contribuyen a la caracterización de este grupo, se presenta una mayor tendencia a los conflictos en las reglas de la relación, específicamente en cuanto a los roles, la distribución de tareas y el manejo de la economía, así como también se presenta una tendencia a mantener una pauta predominante simbiótica. Finalmente, a nivel de la caracterización sociodemográfica este tipo de dinámicas de pareja cuentan con miembros entre los 20 y los 30 años de edad que conviven y cuyas familias tienen conocimiento de su orientación sexual, aunque esto no garantiza el reconocimiento familiar del vínculo.

Como síntesis de las características de este grupo, es posible señalar que se trata de parejas en las que las fuentes de apoyo y validación personal se han reducido para uno de sus miembros, ya sea por motivo de conflictos con la familia de origen o por fuentes de tensión externas, ante lo cual se da un aferramiento emocional con la pareja que genera una fusión en tiempos y actividades y que propicia el escenario para el establecimiento de una pauta predominante simbiótica. Estas relaciones de pareja parecen 
tener un mejor ajuste inicial y se mantienen bajo grandes expectativas emocionales, de apoyo y validación frente a la progresiva fragilización de uno de sus miembros. Luego aparecen los conflictos en las reglas de la relación propios del agotamiento, al no recibir el reconocimiento emocional esperado o al no querer posponer los proyectos personales por mantener la relación de pareja. Finalmente, cuando los conflictos y tensiones se exacerban hasta llevar a dinámicas de maltrato o a amenazas de ruptura, las parejas deciden comenzar un proceso terapéutico, ya que el dilema en el que se encuentran se hace insostenible y experimentan fuertes estados emocionales frente a la necesidad de retomar la propia vida.

\section{Descripción del proceso terapéutico}

En el siguiente apartado, se da cuenta del modelo de abordaje terapéutico desarrollado para la atención clínica a parejas del mismo sexo. En cuanto a la modalidad de atención, en general las parejas que solicitaron asesoría fueron atendidas por los terapeutas por separado, y se tomó la decisión de trabajar con equipo terapéutico en los casos en los que la complejidad de la problemática presentada y de la dinámica conyugal así lo requería, con el fin de velar por el mejor efecto posible de las intervenciones para los/as consultantes.

La estrategia de equipo reflexivo como corresponde dentro de la intervención sistémica se utilizó en los casos en los que resultaba adecuado de acuerdo con las estrategias de intervención planteadas. Inicialmente, se parte de la caracterización general del proceso terapéutico con las seis parejas (Tabla 1), para luego pasar a una descripción de las fases de dicho proceso, en la que se resaltan los elementos comunes y los aspectos diferentes que resultan interesantes.
TABLA 1

Caracterización del proceso terapéutico

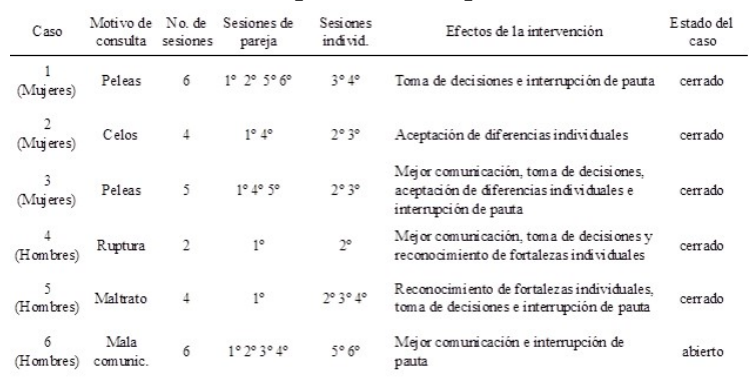

Fuente: elaboración propia.

A continuación, se describen las fases del proceso terapéutico, resaltando los puntos comunes, las diferencias, las intervenciones realizadas y sus efectos sobre las parejas.

(1) Recepción telefónica: en el primer contacto con los miembros de la pareja, el objetivo fue establecer la composición del sistema terapéutico y desarrollar algunas hipótesis iniciales sobre cada caso. En todos los casos, uno de los miembros de la pareja, de mutuo acuerdo con el/la otro/ a, llamó a LIBERARTE para solicitar la cita, reconociendo que tenía un problema de pareja. Se informó sobre las características del servicio, se solicitaron los datos básicos de identificación y remisión, así como una breve descripción del motivo de consulta, y se agendó la cita. Se demandó la asistencia de ambos miembros de la pareja y se evitaron alianzas con alguno de los miembros.

(2) Primera sesión: se llevó a cabo el enganche y la evaluación de la dinámica conyugal, la cual se extendió en los casos N..$^{\circ} 1$ y N. ${ }^{\circ} 6$ a la segunda sesión. Se inició la sesión con una fase social en la que se estableció el rapport con los miembros de la pareja y se amplió la información sobre las características del servicio. Luego se dio paso a la contextualización del caso, indagando acerca de las circunstancias vitales y las condiciones sociales de la pareja y de cada uno de sus miembros y manteniendo la neutralidad terapéutica para evitar responder a las invitaciones de uno o ambos miembros de la pareja a establecer alianzas con los terapeutas, ya que aceptar dichas invitaciones reduce el rango de maniobra de los terapeutas 
y puede contribuir a mantener o incluso a exacerbar la pauta que alimenta el conflicto.

En el caso $N .^{\circ} 5$ fue necesario intervenir directamente para detener la escalada simétrica en la que se encontraba la pareja al llegar a la primera sesión, la cual, debido a la complejidad del caso, amenazaba con llegar a la violencia física y a una exacerbación del conflicto conyugal. En este caso, se tomó la decisión de trabajar con equipo terapéutico, y comenzar entrevistando individualmente a cada uno de los miembros de la pareja durante 20 minutos, con el fin de evaluar su capacidad de contener sus emociones intensas de dolor, rabia y tristeza. A partir de esta evaluación, en la que ambos evidenciaron capacidades de contención de sus emociones y de reflexión sobre lo que les ocurría, así como la explicitación de los propósitos de la primera entrevista conjunta y de las normas de no violencia indispensables para empezar un proceso terapéutico de pareja, se tomó la decisión de proseguir con la entrevista conjunta.

Luego, se exploró la definición del problema de cada uno de los miembros y el proceso de configuración del motivo de consulta. En todos los casos se evidenció que no habían asistido antes a terapia de pareja, a pesar de que su malestar se había originado hacía por lo menos un año. Se indagó acerca de las motivaciones para asistir a la asesoría y acerca de sus expectativas sobre el proceso terapéutico. Esta intervención permitió a la pareja explicitar los temores a ser separados/as por la terapeuta, frente a lo cual se intervino explicitando a los/as consultantes que la continuidad o no de la relación no podía constituirse en el objetivo de la terapia y que esta decisión la tomarían ellos/as a partir de que en el proceso se revisaran las posibilidades de la relación y el alcance del compromiso conyugal. Esta intervención constituyó una primera ampliación de la definición del conflicto presentado por la pareja y facilitó el enganche.

A partir de lo anterior, se llevó a cabo una evaluación de la dinámica de la relación, retomando los cinco ejes mencionados, con el fin de establecer las características del sistema consultante. Se profundizó sobre unas u otras dimensiones, teniendo en cuenta los focos de las tensiones relacionadas con la emergencia del conflicto.

Finalmente, para concluir esta primera sesión se llevó a cabo una redefinición del problema en cuatro de los casos, en la que los terapeutas señalaron a la pareja las conexiones establecidas entre el motivo de consulta, la dinámica de la relación, las necesidades personales y el cambio deseado. Esta intervención corresponde a una conceptualización por parte de los terapeutas, en la que se elaboraron algunas hipótesis relacionales y complejas sobre el conflicto conyugal. En los casos N..$^{\circ} 1$ y N..$^{\circ} 6$ no se contó con elementos suficientes para llevar a cabo la redefinición del problema y por lo tanto se concluyó la sesión con una connotación positiva del conflicto y del manejo del mismo por parte de la pareja. Una intervención final importante en todos los casos implicó dar reconocimiento a los miembros de la pareja por su interés en buscar una solución a su malestar y por comprometerse a expresar lo más abiertamente y tranquilamente posible sus preocupaciones. En el caso $\mathrm{N} .^{\circ} 5$, se llevó a cabo una prescripción paradójica del síntoma en cuanto a pedirles que dejaran al azar la forma como se trataban lanzando una moneda en cada ocasión, con el fin de reafirmar el mensaje de los terapeutas acerca de cómo cada quien debía hacerse cargo de sus emociones.

(3) Sesiones individuales como factor diferenciador: El objetivo de desarrollar las sesiones individuales fue introducir un factor diferenciador en la pareja, que facilitara la ruptura de la pauta simbiótica o ambigua y que permitiera a cada miembro asumir un posicionamiento más claro frente a la relación a través de una exploración más detallada acerca de la propia vida, los proyectos e intereses personales, los motivos para permanecer en la relación, así como las creencias y valores que favorecían la emergencia del conflicto. La historia personal se exploró para clarificar la pauta de relación predominante asociada a la emergencia del conflicto y la participación de cada miembro en dicha pauta. Únicamente, en el caso $\mathrm{N}$. ${ }^{\circ}$ 6 se tomó la decisión de llevar a cabo cuatro 
sesiones conjuntas antes de proponer las sesiones individuales, en tanto en este caso el motivo de consulta, referido a la mala comunicación, y las dificultades evidenciadas a partir de una pauta comunicacional de evitación del conflicto y de un distanciamiento progresivo implicaban la necesidad de que ambos miembros de la pareja estuvieran presentes para abordar las distintas dimensiones que mantenían y amplificaban los conflictos.

Por otra parte, en el caso $\mathrm{N}^{\circ} 5$ se llevaron a cabo estas sesiones individuales con equipo terapéutico, y en ellas se utilizó la intervención del equipo reflexivo, con el fin de devolver a los consultantes la lectura que se hacía de su situación emocional y de la forma como a través de su historia se daba cuenta de la pauta ambigua que mantenían en la relación. El efecto de esta intervención fue el posicionamiento de ambos consultantes frente a la propia vida y a sus elecciones frente a sus preferencias, ante lo cual se dio una ruptura acordada entre ellos y uno de ellos decidió continuar con un proceso terapéutico individual para asumir dicha ruptura.

(4) Devolución de la lectura de los terapeutas y posicionamiento de los miembros de la pareja: Luego de las sesiones individuales se llevó a cabo en cuatro de los casos una sesión conjunta en la que se hizo una devolución por parte de los terapeutas acerca de la lectura del caso y en la que se retomó, a modo de relato, la referencia a las dimensiones de la dinámica de la relación evaluadas en las sesiones anteriores para evidenciar el dilema de cada una. En el caso N. ${ }^{\circ}$ 1, se llevó a cabo la intervención de equipo reflexivo, a partir de la cual se describió a la pareja la pauta simbiótica que manejaban y que dificultaba la toma de postura por parte de ambas. Esta intervención tuvo el efecto de facilitar dicha toma de postura y de permitir la subsiguiente exploración de las posibilidades de la relación.

(5) Exploración de las posibilidades de la relación: La fase final del proceso terapéutico consistió en explorar las posibilidades de la relación bajo unas condiciones y necesidades personales que se fueron clarificando a lo largo de la terapia. Se tuvieron en cuenta los cambios mínimos evidenciados a lo largo del proceso como indicador valioso sobre las posibilidades de cambio en la dinámica relacional. El efecto de esta intervención, en la que se indagó sobre los efectos del proceso sobre cada miembro y sobre la relación, fue la explicitación de las alternativas de cambio que ya se habían empezado a llevar a cabo o que se podían concretar a partir de ese momento, en tanto las cuatro parejas con las que se llegó a esta fase se reafirmaron en considerar la relación que mantenían como su mejor opción. Es así como los terapeutas hicieron sugerencias concretas para el mantenimiento de los cambios evidenciados y les dieron el reconocimiento sobre los logros y las claridades obtenidas.

\section{Discusión}

Retomando los objetivos de este estudio, se evidencia que fue posible identificar las pautas de relación que configuran como mínimo dos tipos de parejas con características predominantes distintas, y que se logró elaborar un modelo de intervención para parejas del mismo sexo con criterios clínicos y efectos específicos.

\section{Implicaciones conceptuales}

Una primera implicación en este sentido se relaciona con la necesidad de llenar un vacío en el conocimiento científico alrededor de las dinámicas de relación de parejas del mismo sexo, señalado por McNair y Spitalnick (2005). Los resultados obtenidos permiten tener una mirada compleja y ecosistémica acerca de las características predominantes de dichas parejas para dar cuenta de sus particularidades y proponer intervenciones ajustadas que activen las posibilidades de cambio dentro de los procesos terapéuticos, conectando las diferentes dimensiones de la dinámica conyugal con las características sociodemográficas relevantes, los motivos de consulta y las pautas de relación más amplias.

Esta perspectiva evita los riesgos de fragmentar las dinámicas de relación de dichas parejas a partir de referentes explicativos parciales 
o heteronormativos, ya que es un error igual de serio el suponer que las dificultades presentadas como motivo de consulta se deben exclusivamente a la orientación sexual que suponer que estas no tienen relación alguna con la orientación sexual (McNair \& Spitalnick, 2005). En esta investigación, la orientación sexual se abordó no como una variable entre otras, sino como una condición subjetiva, interaccional y contextual con implicaciones específicas en las dinámicas y los conflictos conyugales. Estas conexiones son útiles, pues en abordajes anteriores se le ha dado peso a una de estas dimensiones, desconociendo o adjudicando una menor relevancia a las otras dos, lo cual ha llevado a conceptualizaciones fragmentadas, individualistas o que pierden de vista la dimensión subjetiva, privilegiando el impacto de la dimensión contextual sobre las dinámicas de pareja (Fraenkel \& Gurman, 2002).

Desde el marco epistemológico constructivista, resulta fundamental rescatar la importancia de la dimensión individual comprendida a partir de un enfoque ecosistémico, referida a la vivencia subjetiva de la propia sexualidad, así como a los marcos de referencia, los motivos para permanecer en la relación y las fuentes de tensión de cada miembro de la pareja, en tanto permite una comprensión amplia de la configuración de la relación y reconoce a cada persona desde su subjetividad en cuanto a sus posturas, experiencias, cuestionamientos, conflictos y convicciones.

Teniendo en cuenta las nuevas garantías legales para parejas del mismo sexo en Colombia a partir de las Sentencias de la Corte Constitucional (Colombia Diversa, 2016a) y los cambios culturales que remarcan los Derechos Humanos asociados a la diversidad sexual, la referencia a la dimensión individual conectada con las dimensiones contextuales e interaccionales particulares rescata la dimensión psicológica de la vivencia subjetiva y relacional de la diversidad sexual, para evitar caer en comprensiones exclusivamente centradas en los marcos legales.
A partir de lo anterior, vale la pena resaltar algunas de las precisiones conceptuales desarrolladas en este estudio que tienen implicaciones importantes para las conceptualizaciones vigentes alrededor de las parejas del mismo sexo. En primera medida, la caracterización sociodemográfica de estas parejas, en cuanto a condiciones de edad, convivencia en pareja y conocimiento familiar de la orientación sexual que se asocian con la emergencia del conflicto conyugal, no ha sido abordada de forma específica en estudios anteriores, ya que las referencias previas únicamente dan cuenta de la importancia de la relación de pareja como motivo de consulta a nivel clínico en personas sexualmente diversas (Connolly, 2004) o establecen asociaciones parciales entre el sexo de los miembros de la pareja y los conflictos interaccionales más frecuentes (McNair \& Spitalnik, 2005; Ossana, 2000; Sandfort \& De Keizer, 2001).

Por otra parte, el aporte de este estudio implica una caracterización general de los dos grupos de parejas con pautas de relación predominantes distintas, lo cual permite un reconocimiento de la dimensión interaccional. Las hipótesis de los investigadores sobre la forma como emerge y se mantiene la pauta simbiótica en un grupo importante de parejas del mismo sexo se sustenta, en primera medida, en dimensiones contextuales, en las que se destaca la presencia marcada de conflictos con las familias de origen y de una falta de legitimación de la relación a nivel familiar y social; en segunda medida, en la vivencia subjetiva de la propia sexualidad, específicamente en cuanto a la forma como cada miembro de la pareja elabora dichos conflictos con otros y asume un estilo relacional de aislamiento de otras relaciones significativas como forma de evitar el rechazo o la discriminación y en tercera medida, en el mutuo encaje en la dinámica conyugal de las necesidades emocionales de sus miembros, sobre lo cual se sustenta el vínculo.

Por tanto, las conclusiones a las que llegan investigadores como McNair y Spitalnik (2005) sobre la mayor presencia de pautas simbióticas en parejas de mujeres no corresponden con lo 
que se concluye en este estudio, ya que, tanto parejas de hombres, como las de mujeres, pueden presentarlas y son otras precisiones las que dan cuenta de la emergencia de procesos simbióticos.

Precisamente, uno de los hallazgos más importantes en este sentido invita a poner en cuestionamiento algunas de las asociaciones previas, como las desarrolladas por Blumenstein y Schwartz (1983), Boyle y Cove (2002) y Sandfort y De Keizer (2001), que se fundamentan en estereotipos de género como la idea de que son las parejas de hombres las que presentan más dificultades en la vida sexual y las de mujeres las que tienen mayores insatisfacciones en el campo afectivo. Dichas generalizaciones desconocen los múltiples aspectos que entran en juego a la hora de explicar los conflictos de las parejas del mismo sexo, ya que parejas en conflicto de hombres y de mujeres tienen en su mayoría insatisfacciones en la vida sexual y en sus expectativas afectivas, especialmente en cuanto a la exclusividad del vínculo.

Por otra parte, la pauta ambigua no se encuentra definida en la literatura científica y constituye uno de los aportes importantes de este estudio, pues su descripción permite explicar las dinámicas de maltrato y chantaje emocional desde una lógica coherente que da cuenta de la configuración confusa de estas relaciones y los conflictos individuales con la orientación sexual propia a partir de valores heteronormativos y de experiencias heterosexuales previas, ante lo cual la definición de la relación y el posicionamiento de los miembros no es claro ni se plantean las propias necesidades emocionales abiertamente, lo cual se hace insostenible a tal punto que se favorecen dinámicas violentas al interior de la pareja.

Esta descripción de la pauta ambigua resulta útil, en tanto la violencia al interior de las parejas del mismo sexo es aún un tema poco abordado en el ámbito científico, social y específicamente en el movimiento LGBT, pues se teme que desde miradas heterocentristas que condenan la homosexualidad o que descalifican la legitimidad de estas afiliaciones amorosas dicha constatación pueda llevar a interpretaciones patologizantes sobre ellas. Sin embargo, la mirada de los investigadores no está fundamentada en un criterio de normalidad para valorar su legitimidad y se considera que el reconocimiento de los dilemas que viven es precisamente el primer paso para trascender las polaridades entre versiones románticas vs. versiones satanizadas y poder atender sus necesidades específicas.

Finalmente, la apuesta por unos ejes de evaluación clínica de las dinámicas de dichas parejas que avancen más allá del manejo de un lenguaje no homofóbico, de la legitimación de estas afiliaciones amorosas o del reconocimiento de sus recursos para enfrentar los retos sociales (Hamrin et al., 2006; Herek, 2006; Ossana, 2000; Slater, 1999), resulta novedosa e implica rigurosidad conceptual en este tema aún controversial al asumir referentes clínicos claros.

En este estudio, no se llevó a cabo una comparación directa entre parejas del mismo sexo y parejas heterosexuales, pero se evidencia que existen referentes comunes a todas las modalidades de vinculación humana (tales como el intercambio afectivo y sexual, la comunicación, las reglas de la relación, el manejo del poder y la relación con el entorno), lo cual no implica que las dinámicas sean necesariamente idénticas en ambos tipos de parejas. Las principales distinciones corresponden al peso de ciertas dimensiones en la consolidación de las relaciones de pareja entre personas del mismo sexo, así como en la emergencia de los conflictos conyugales.

Por ejemplo, la dimensión de las relaciones con la familia de origen es común a cualquier forma de vinculación, pero la marcada tendencia a la falta de legitimación del estatus de pareja por parte de las familias de origen y su asociación a los procesos simbióticos, a las tensiones alrededor de los conflictos con la orientación sexual propia o de la pareja y finalmente su asociación general a la emergencia del conflicto aparecen como propias de parejas del mismo sexo. Por otra parte, la dimensión relacionada con el marco de referencia individual también es común a cualquier pareja, pero el impacto de las creencias heteronormativas y de la experiencia subjetiva referida a la homofobia internalizada sobre las dinámicas de la pareja es diferencial en parejas 
del mismo sexo, ya que la validación social y personal del vínculo de pareja es cuestionada constantemente y los referentes alternativos que validen la propia experiencia de pareja no se encuentran fácilmente. Finalmente, la ausencia de prácticas que formalicen la relación en parejas del mismo sexo y la respuesta de estas en cuanto a una convivencia casi inmediata luego de conocerse, así como las inseguridades desde el inicio, la tendencia a mantener triángulos en la relación y la asunción de identidades discretas favorecen la confusión en la definición de la relación y, por tanto, adquieren peso en la configuración de la relación y las vicisitudes en parejas del mismo sexo.

\section{Implicaciones para el abordaje terapéutico}

Desde una mirada compleja y ecosistémica, en la que los criterios para evaluar el cambio y para estructurar las estrategias de intervención no se fundamentan únicamente en las respuestas emocionales de agrado o frustración de los/ as consultantes ni en el alivio paliativo del sufrimiento o la remisión de los síntomas, sino que se privilegia un cambio de segundo orden a partir de una explicación hipotética de las pautas de interacción más amplias que favorecen la emergencia del conflicto conyugal (Bateson, 1976), la finalidad clínica del proceso terapéutico es favorecer una mayor autonomía responsable y diferenciación en los/as consultantes frente a sus necesidades, preferencias y opciones vitales, incluyendo la pareja, con el fin de facilitar un posicionamiento individual más claro y favorecer la ruptura de las pautas simbióticas o ambiguas. En este sentido, la intervención referida a plantear sesiones individuales con los miembros de la pareja como factor diferenciador, se sustenta bajo el criterio de poder explorar las creencias, motivos, preferencias y vulnerabilidades de cada miembro de la pareja para lograr un posicionamiento personal, a partir de la confrontación de dichos aspectos individuales que en presencia de la pareja no se explicitan debido al temor frente a cualquier amenaza de ruptura de la relación.
Esto se corresponde también con los principales efectos de la intervención evidenciados en cuanto a la toma de decisiones frente a la continuidad de la relación de pareja, el reconocimiento de las fortalezas y la aceptación de las diferencias individuales, así como la interrupción de la pauta predominante de la relación.

Partiendo de estas premisas propias de un enfoque estratégico (Haley, 1963), el modelo de abordaje propuesto para el trabajo terapéutico con parejas del mismo sexo parte de criterios clínicos claros que mantienen como eje central la dimensión psicológica en cuanto a llevar a cabo una evaluación completa y precisa de la dinámica conyugal siguiendo los ejes definidos.

Este punto resulta importante, en tanto diversos autores como McNair y Spitalnick (2005) y Connolly (2004) reconocen la falta de entrenamiento específico de los/as terapeutas para el abordaje de parejas del mismo sexo y el vacío en cuanto a investigaciones que revisen la utilidad de diferentes enfoques terapéuticos para el trabajo con estas parejas. Esta propuesta de abordaje contribuye precisamente a clarificar los criterios que pueden guiar un acercamiento fundamentado que no pretenda acomodar las propuestas útiles con parejas heterosexuales a parejas del mismo sexo ni sustentarse únicamente en la validación de la diversidad sexual.

Por otra parte, al tratarse de terapia de pareja, el objeto de la intervención es la relación y no solo sus miembros, por lo cual se enfatiza sobre la necesidad de que ambos/as tengan conocimiento del proceso terapéutico a iniciar e idealmente asistan a la primera entrevista de pareja, para así diseñar conjuntamente las estrategias de intervención más ajustadas a sus necesidades, pues aceptar que desde el inicio de la terapia solo uno de los miembros asista a las sesiones implica correr un mayor riesgo de ser triangulado en la dinámica mantenedora de los conflictos y movilizar el sistema con poca claridad de las implicaciones de los cambios propuestos. Sin embargo, es posible ajustar la propuesta de intervención a las particularidades de cada caso, bajo argumentos clínicos claros que permitan 
una estructuración estratégica y responsable del sistema de ayuda.

A su vez, los criterios de los investigadores para la elaboración de la redefinición del problema estuvieron referidos a establecer conexiones hipotéticas entre el motivo de consulta, la configuración de la relación y sus vicisitudes, los motivos para permanecer en la relación de cada uno de los miembros y las fuentes de tensión que han tenido mayores impactos en la pauta predominante, para finalmente articular las explicaciones sobre los focos de conflicto en pautas relacionales más amplias que explican de forma compleja la emergencia de los conflictos interaccionales presentados. Finalmente, el criterio de los investigadores/ terapeutas para la utilización de la técnica de equipo reflexivo como corresponde dentro de la intervención sistémica (Andersen, 1994) parte de la observación de la complejidad de algunos casos y del interés de los terapeutas por velar por el mejor efecto posible de las intervenciones. A partir de lo anterior, resulta importante señalar que desde esta propuesta el espacio terapéutico no puede convertirse en un espacio ideologizante, sino que por el contrario debe permitir a los/ as consultantes el ejercicio de su autonomía en la definición y en la vivencia de su sexualidad, sin que necesariamente esta deba inscribirse o encasillarse según rótulos o pertenencia a grupos sociales determinados. En este sentido, surgen cuestionamientos importantes frente a propuestas como la terapia afirmativa para homosexuales, según la cual lo más útil para el trabajo con personas homosexuales y parejas del mismo sexo es no problematizar la orientación sexual y enfatizar en que los/as consultantes afirmen su identidad homosexual para lograr un mayor bienestar (Ardila, 2007). Desde el modelo de abordaje presentado en este estudio, se comparte la importancia que tiene el posicionamiento del sujeto y en consecuencia su posibilidad de tomar decisiones autónomas en los diferentes ámbitos de su vida, incluyendo el ámbito de la pareja, pero no se considera que dicho posicionamiento tenga que ir dirigido a la definición permanente de la orientación sexual propia según rótulos que limitan su autonomía y desconocen las diversidades y las múltiples vivencias subjetivas de la orientación sexual.

Tampoco se podría afirmar que el criterio clínico que conecta las particularidades interaccionales, subjetivas y contextuales puede sustituirse por una hipótesis lineal en la que se considere que el bienestar o malestar de los consultantes depende de que se reafirmen en su identidad homosexual, en tanto la identidad se concibe como una abstracción analítica de una vivencia relacional matizada y cambiante a lo largo de la vida. Por lo tanto, la labor terapéutica debe estar dirigida a promover las exploraciones que en este sentido decidan hacer los/as consultantes y a ampliar sus posibilidades de autonomía responsable.

Por último, se consideran válidos los argumentos presentados por Gurman (2001) acerca de la redundancia de hablar de terapia de pareja breve, en tanto la duración de los procesos terapéuticos no superó las 12 sesiones y se logró la interrupción de la pauta en los casos en los que se llevaron a cabo de 8 a 12 sesiones. Por tanto, resulta importante acoger los principios de economía, parsimonia, el enfoque en las fortalezas de la pareja y el énfasis en el presente para proponer un modelo de abordaje eficiente, en el que las historias personales y conjuntas se tienen en cuenta para clarificar las pautas y el énfasis se pone en las elaboraciones que comprometen las distintas dimensiones que movilizan el cambio.

\section{Implicaciones sociales}

Las implicaciones que puede tener este estudio para personas sexualmente diversas hacen referencia a la posibilidad de recibir una atención psicológica en la que se reconocen las particularidades de las condiciones de vida, las dinámicas de relación y las vivencias subjetivas alrededor de la sexualidad, teniendo conceptualizaciones que se fundamentan en un marco epistemológico, teórico y metodológico congruente para dar respuesta a sus demandas de ayuda. 
Más ampliamente, el reconocimiento de la existencia de conflictos conyugales que se exacerban hasta llegar a pautas violentas o de chantaje emocional puede sumar elementos para el desarrollo de propuestas individuales, familiares y colectivas que trasciendan las garantías legales logradas hasta el momento y que aborden más directamente los focos de conflicto para lograr cambios desde distintas instancias que desbordan la atención clínica y que requieren de la participación de otras disciplinas.

Las implicaciones para profesionales de la psicología y de otras disciplinas del campo de la salud son claras, al tener en cuenta el vacío en el conocimiento científico alrededor de las dinámicas de relación de parejas del mismo sexo y la consecuente falta de entrenamiento específico para el trabajo con estas parejas. Este estudio permite avanzar en las observaciones y asociaciones más generales en torno a las dinámicas de las parejas en general, aportando precisiones y puntos a tener en cuenta desde lo evidenciado para los casos estudiados. Aunque no se pretende llevar a cabo generalizaciones de los resultados obtenidos, las conexiones y elaboraciones realizadas sí pueden constituir guías de acercamiento a la comprensión y al abordaje de estos casos, las cuales estarán matizadas por las particularidades que se evidencien. En conclusión, este estudio contribuye a la transformación de prácticas clínicas excluyentes y discriminatorias que intentan comprender e intervenir en todas las parejas con el mismo lente.

Finalmente, las implicaciones para la comunidad en general tienen que ver con la responsabilidad compartida de visibilizar lo que ha sido invisibilizado por mucho tiempo en cuanto a la diversidad sexual y a las afiliaciones amorosas entre personas del mismo sexo. Es así como las implicaciones de este estudio hacen referencia a la necesidad que como comunidad se tiene de reconocer y potenciar la diversidad humana, no solo en cuanto a la sexualidad, sino en todas las dimensiones de la vida, para lograr un ejercicio autónomo y responsable de la ciudadanía.

\section{Implicaciones metodológicas}

Las implicaciones metodológicas de este estudio hacen referencia principalmente a la búsqueda de solidez y rigor conceptual, lo cual se conecta con la coherencia a lo largo de la investigación-intervención desde las premisas del pensamiento sistémico y desde la práctica clínica e investigativa, resultando en ejes comprensivos que se fueron precisando a partir de los resultados obtenidos en los distintos análisis. La apuesta por un diseño de investigación-intervención permitió establecer conexiones importantes y ajustadas entre las conceptualizaciones teóricas, los criterios clínicos y las prácticas interventivas. La aproximación metodológica mixta permitió desarrollar comprensiones nutridas de diferentes tipos de análisis, y la mirada cualitativa a los datos cuantitativos, la reconstrucción de las pautas relacionales más amplias a partir de las asociaciones significativas a nivel estadístico, lo cual constituye una invitación a cuestionar la polaridad metodológica entre los estudios cuantitativos y los cualitativos bajo criterios de pragmatismo y de coherencia con la perspectiva epistemológica asumida.

Finalmente, las limitaciones en este estudio hacen referencia a que los/as participantes se eligieron bajo un muestreo por conveniencia, ante lo cual no se tuvieron en cuenta parejas que viven en lugares del país distintos a Bogotá. Por otra parte, en el abordaje terapéutico con las parejas no se tuvo la posibilidad de hacer un seguimiento a largo plazo para evaluar la perdurabilidad de los efectos de la intervención evidenciados debido al tiempo delimitado para el estudio.

\section{Agradecimientos}

Este artículo de investigación resulta del trabajo de grado de la maestría en psicología clínica en la Pontificia Universidad Javeriana titulado "Comprensión de las dinámicas de relación de parejas del mismo sexo a partir de la intervención clínica sistémica", elaborado en 2010-2011 por Carolina Herrera Small y Simón Torres Orozco 
de LIBERARTE bajo la dirección de Ángela Hernández Córdoba, Ph.D.

\section{Referencias}

Andersen, T. (1994). El equipo reflexivo: diálogos y diálogos sobre los diálogos. Barcelona: Gedisa.

Andrews, B., \& Long, J. (2007). Fostering strength and resiliency in same-sex couples. Journal of Couple $\mathcal{E}$ Relationship Therapy, 6(1), 153-165.

Ardila, R. (2007). Terapia afirmativa para homosexuales y lesbianas. Revista Colombiana de Psiquiatría, 36(1), 67-77.

Asociación de Psicología de Puerto Rico. (2008). Estándares para el trabajo e intervención en comunidades lesbianas, gay, bisexuales y e identidades trans (LGBT). San Juan, PR: Autor. Recuperado de http://www.asppr.ne t/pdf/LGBT.pdf

Beougher, S., Chakravarty, D., Darbes, L., Hoff, C., \& Nelands, T. (2010). Relationship characteristics and motivations behind agreements among gay male couples: Differences by agreement type and couple serostatus. AIDS Care 22 (7), 827-835.

Bateson, G. (1976). Pasos hacia una ecología de la mente. Buenos Aires: Carlos Lohlé.

Blair, K., \& Holmberg, D. (2009). Sexual desire, communication, satisfaction, and preferences of men and women in same-sex versus mixed-sex relationships. The Journal of Sex Research, 46(1), 57-66.

Blumenstein, P., \& Schwartz, P. (1983). American couples: Money, work, and sex. Nueva York: Morrow.

Boyle, M., \& Cove, J. (2002). Gay men's self-defined sexual problems, perceived causes and factors in remission. Sexual and Relationship Therapy, 17(3), 137-147.

Brodnicki, C., Couch, R., Dudley, M., Duhigg, J., Riggle, E., \& Rostosky, S. (2005). Same-sex couples' experiences with homonegativity. Journal of GLBT Family Studies, 1(4), 61-78.

Brown, M. \& Woollams, S. (1989). Transactional analysis: A modern and comprehensive text of
TA theory and practice. Dexter, MI: Huron Valley Institute.

Colombia Diversa. (2016a). Asuntos legales LGBT. Recuperado de http://colombiadive rsa.org/colombiadiversa/index.php/publica ciones/asuntos-legales-lgbt

Colombia Diversa. (2016b). Informes de derechos humanos. Recuperado de http://colombiadiversa.org/colombiadiv ersa/index.php/informes-de-derechos-hum anos

Connolly, C. (2004). Clinical issues with samesex couples. Journal of Couple E Relationship Therapy, 3(2-3), 3-12.

Diemer, M., Mackey, R., \& O’Brien, B. (2004). Relational factors in understanding satisfaction in the lasting relationships of same-sex and heterosexual couples. Journal of Homosexuality, 47(1), 111-136.

Fraenkel, P., \& Gurman, A. (2002). The History of couple therapy: A millennial review. Family Process, 41 (2), 199-260.

Frederickson, B., Gottman, J., Gross, J., Levenson, R., McCoy, K., Rosenthal, L., ... Yoshimoto, D. (2003). Journal of Homosexuality, 45(1), 23-43.

García, A. (2009). Tacones, siliconas, hormonas y otras críticas al sistema sexo-género. Revista Colombiana de Antropología, 45(1), 119-146.

Gottman, J., Levenson, R., Swanson, C., Swanson, K., Tyson, R., \& Yoshimoto, D. (2003). Observing gay, lesbian and heterosexual couples' relationships. Journal of Homosexuality, 45 (1), 65-91.

Gurman, A. (2001). Brief therapy and family/ couple therapy: An essential redundancy. Clinical Psychology: Science and Practice, 8(3), 51-65.

Haley, J. (1963). Estrategias en psicoterapia. Barcelona: Toray.

Hamrin, R., Prather, R., Riggle, E., \& Rostosky, S. (2006). The execution of legal documents by sexual minority individuals. Journal of Counseling Psychology, 53(1(), 48-56.

Herek, G. (2006). Legal recognition of same-sex relationships in the United States: A social 
science perspective. American Psychologist, 61(6), 607-621.

Hernández, A. (2004). Psicoterapia sistémica breve: la construcción del cambio con individuos, parejas y familias. Bogotá: Editorial El Buho Ltda.

International Lesbian, Gay, Bisexual, Trans and Intersex Association. (2015). State sponsored homophobia 2015: A world survey of laws: criminalisation, protection and recognition of same-sex love. Ginebra: Autor.

Maturana, H. (1995). La realidad objetiva o construida. Madrid: Anthropos.

McNair, L., \& Spitalnick, J. (2005). Couples therapy with gay and lesbian clients: An analysis of important clinical issues. Journal of Sex E Marital Therapy, 31 (3), 43-56.

Ossana, S. (2000). Relationship and couples counseling. En K. Bieschke, R. Perez \& K. DeBord (Eds.), Handbook of counseling and psychotherapy with lesbian, gay, and bisexual clients (pp. 275-302). Washington: American Psychological Association.

Sanders, G. (2009). Men together: Working with gay couples in contemporary times. En P. Papp (Ed.), Couples on the fault line directions for therapists (pp. 222-256). Nueva York: Guilford Press. Recuperado de http://www.familytherapy.org/document s/gaycouples.PDF

Sandfort, T., \& De Keizer, M. (2001). Sexual problems in gay men: An overview of empirical research. Annual Review of Sex Research, 12(2), 93-120.

Slater, S. (1999). The lesbian family life cycle. Nueva York: Free Press.

\section{Notas}

* Artículo de investigación.

[1] Organización de asesoría psicológica para personas sexualmente diversas con sede en Bogotá, Colombia. 